Modeling, Identification and Control, Vol. 33, No. 1, 2012, pp. 13-25, ISSN 1890-1328

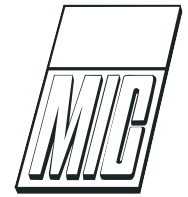

\title{
Optimization of lift gas allocation in a gas lifted oil field as non-linear optimization problem
}

\author{
Roshan Sharma $^{1}$ Kjetil Fjalestad $^{2}$ Bjørn Glemmestad ${ }^{1}$
}

\author{
${ }^{1}$ Department of Electrical, Information Technology and Cybernetics, Telemark University College, Porsgrunn, Nor- \\ way. E-mail: \{roshan.sharma,bjorn.glemmestad\}@hit.no \\ ${ }^{2}$ Statoil Research Center, Porsgrunn, Norway. E-mail: kfja@statoil.com
}

\begin{abstract}
Proper allocation and distribution of lift gas is necessary for maximizing total oil production from a field with gas lifted oil wells. When the supply of the lift gas is limited, the total available gas should be optimally distributed among the oil wells of the field such that the total production of oil from the field is maximized. This paper describes a non-linear optimization problem with constraints associated with the optimal distribution of the lift gas. A non-linear objective function is developed using a simple dynamic model of the oil field where the decision variables represent the lift gas flow rate set points of each oil well of the field. The lift gas optimization problem is solved using the 'fmincon' solver found in MATLAB. As an alternative and for verification, hill climbing method is utilized for solving the optimization problem. Using both of these methods, it has been shown that after optimization, the total oil production is increased by about $4 \%$. For multiple oil wells sharing lift gas from a common source, a cascade control strategy along with a nonlinear steady state optimizer behaves as a self-optimizing control structure when the total supply of lift gas is assumed to be the only input disturbance present in the process. Simulation results show that repeated optimization performed after the first time optimization under the presence of the input disturbance has no effect in the total oil production.
\end{abstract}

Keywords: Optimization, non-linear programming, cascade control structure, gas lifted oil well, hill climbing, self-optimization

\section{INTRODUCTION}

For a gas lifted oil field where multiple oil wells share the lift gas supplied by the common source (see Figure 1), proper distribution of the available gas is an important issue for maximizing the total oil produced from the oil wells. The set points for the mass flow rate of the lift gas for each of the gas lift choke valves have to be allocated in a way that the distribution yields maximum oil production using the available gas. Stable or steady operation does not guarantee optimal operation i.e. even though the field is operating in a stable manner, the lift gas might have been distributed among the oil wells in a non-optimal way and the wells might have been producing less than what they can actually produce.

For the Norne oil field in the North Sea with five gas lifted oil wells, the objective is to distribute the available gas ensuring optimal production of oil. The amount of lift gas available is assumed to be limited. Thus, optimization for the oil field for this case is the task of finding out the optimal set points of the five flow controllers. Each flow controller controls the opening and closing of the gas lift choke valve of each well. In other words, the decision variables for the optimization problem are the nominal set points of the flow controllers. Using these optimal set points for the gas mass flow rate of the lift gas, the total oil produced from the five 


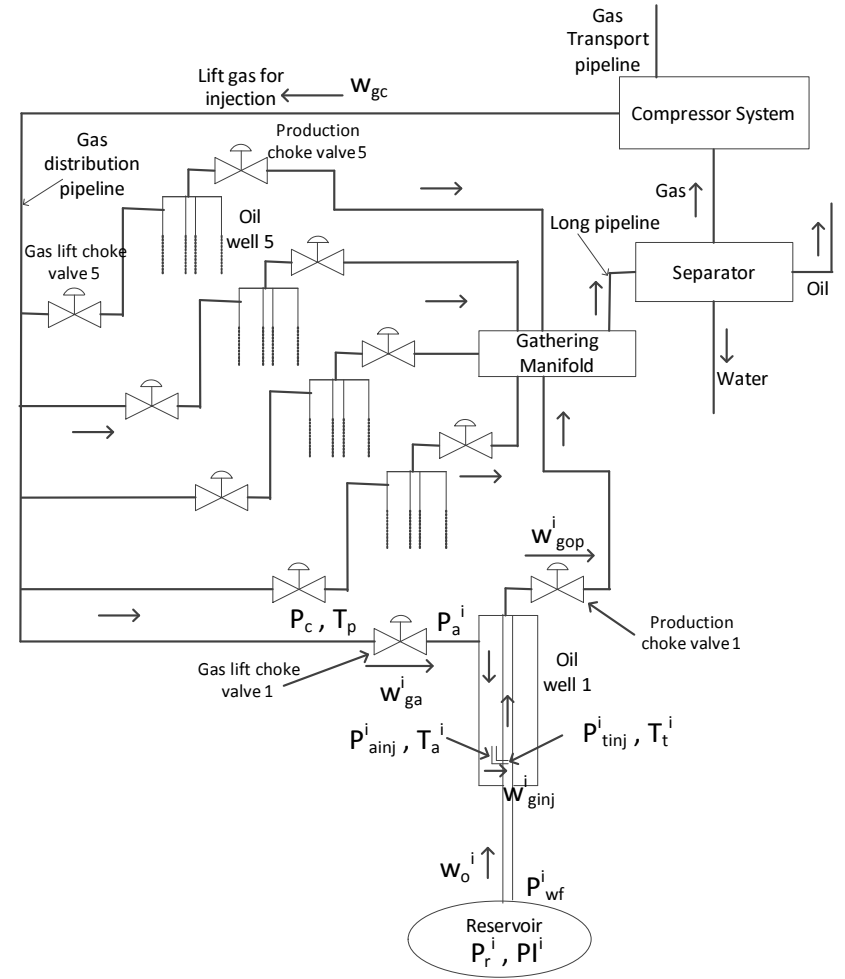

Figure 1: Schematic of an oil field with five oil wells

oil wells should be maximized using the limited amount of gas available.

A non-linear gas lift optimization problem has been formulated using a simple model of the oil field. A brief explanation of the model of the oil field is given in Section 2. Section 3 contains details about the development of the non-linear objective function with constraints. One of the objectives of the paper is to solve this non-linear optimization problem using the 'fmincon' solver of the MATLAB Optimization toolbox as described in Section 4. In addition, the optimization problem has also been solved using hill climbing method as described in Section 5. The focus of this paper is to show through simulation results that both the optimization methods cause an increase in the total oil production from the field. Comparison of the two optimization methods is described in Section 6 .

To control the gas flow rates and the pressure of the common gas distribution pipeline, four control structures were proposed by Sharma et al. (2011) for the same oil field. In this paper, a steady state optimizer working along with the cascade control structure is developed. The optimizer loop rests on top of the control loop and provides the optimal set points to the control loop as shown in Figure 2. The combined structure functions as a self-optimizing control scheme as described in Section 4.3.

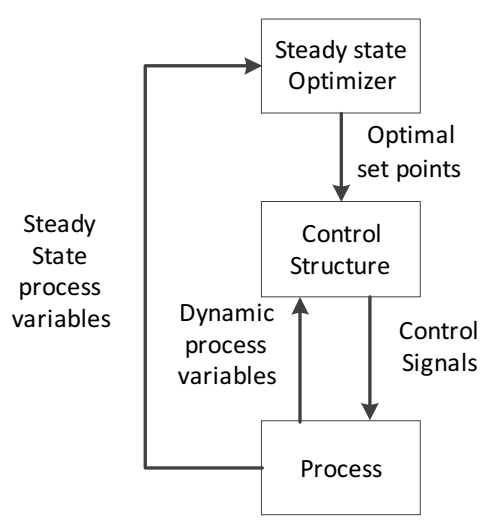

Figure 2: Optimization hierarchy

Optimization of gas lifted oil field has been a research of interest to many authors. Penalty function or Sequential Unconstrained Minimization Technique (SUMT) which can accommodate both the equality constraints and inequality constraints needed to solve the non-linear optimization model of the gas allocation to a gas lifted oil field was proposed by Zhong et al. (2004). Daily well scheduling in gas lifted petroleum fields has been formulated and solved by using mixed integer nonlinear (MINLP) model (Kosmidis et al., 2005) where the discrete decisions include the operational status of wells, the allocation of wells to manifolds or separators and the allocation of flow lines to separators, and the continuous decision include the well oil rates and the allocation of gas-togas lift wells. Dynamic programming has been be used for solving a gas-lift optimization problem (Camponogara and Nakashima, 2006) where the gas-lift optimization problem can be casted as a mixed integer nonlinear programming problem whose integer variables decide which oil well should produce, while the continuous variables allocate the gas-compressing capacity to the active ones. Computational scheme using genetic algorithm has been used to find optimum gas injection rate (Saepudin et al., 2007; Ray and Sarker, 2007) for gas lifted oil field and also for dual gas lift system (Sukarno et al., 2009). For gas lift optimization, a high dimensional problem has been reduced into one single variable problem by using Newton reduction method based on upper convex profile (Rashid, 2010). Gas-lift optimization has been formulated and solved by using an objective function considering the annualized capital costs on compressor, turbine and gas pipelines, the operating costs related to fuel and the revenue from produced oil (Souza et al., 2010). 


\section{MODEL OF THE OIL FIELD}

A simple model of a gas lifted oil well where all the necessary and important components are taken into account is shown in Figure 3. The oil field consists of five gas lifted oil wells which share the lift gas from the common distribution manifold. Figure 1 shows a schematic of the oil field with five oil wells. In this paper, the details about the development of the model of the oil field have not been discussed and only the description of the oil field is included. The model proposed by Sharma et al. (2011) has been used here in this paper.

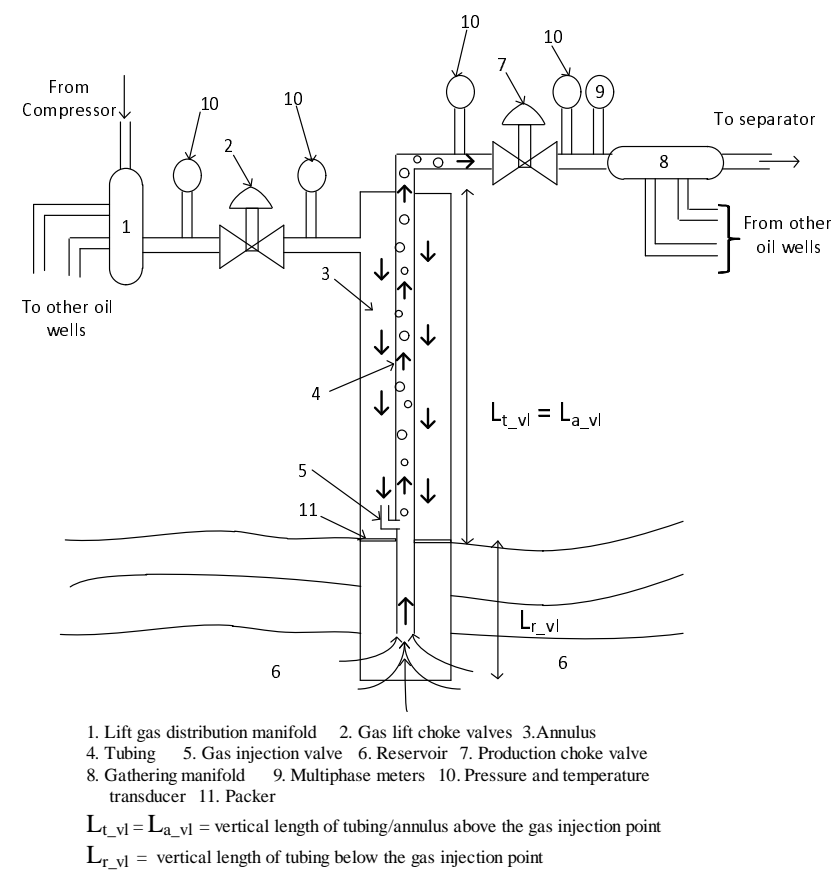

Figure 3: Different components of a gas lifted oil well

The compressor outputs a highly pressurized lift gas into the gas distribution pipeline. The lift gas enters into the annulus of each well from this common gas distribution manifold. The amount of lift gas to be injected into each well is controlled by the gas lift choke valve present in the well head of each oil well.

From the annulus, the high pressure lift gas is injected into the tubing at a proper depth through the gas injection valve (see Figure 3 ). The gas injection valve is designed in a way that the back flow of fluid into the annulus from the tubing does not occur through it.

The injected gas mixes with the multiphase fluid (crude oil, water and gas produced from the reservoir) in the tubing at the point of injection thereby reducing its density and the weight of the liquid column in the tubing. This causes the differential pressure be- tween the reservoir ( $P_{r}^{i}$, superscript ' $i$ ' represents the $i^{\text {th }}$ oil well for all symbols used in this paper) and bottom hole pressure $\left(P_{w f}^{i}\right)$ to be increased causing the liquid column to flow upwards to the surface. The production choke valve controls the flow rate of the fluid $\left(w_{\text {gop }}^{i}\right)$ produced from the reservoir and is assumed to be $100 \%$ fully open. The mixture of the gas, water and oil flowing out of each of the wells through the production choke valves is collected together in the common gathering manifold and finally transported to the separator where they are separated into their respective constituents. The gas is then sent back to the compressor system and recycled to be used for lifting purpose.

Pressure and temperature transducers measure the pressure and temperature both downstream and upstream of the production choke valve and gas lift choke valve. A multiphase flow meter is installed downstream of the production choke valve and is used to measure the flow rate of oil, gas and water individually. The packer is used to seal the bottom of the casing annulus, which funnels all of the production into the tubing string, so all of the available gas energy is utilized to lift the fluid.

For the purpose of gas injection distribution, control and optimization, friction losses have not been taken into account. All phases of the multiphase fluid are assumed to be evenly distributed with no slugging. The temperature of lift gas and the multiphase fluid in all sections of pipeline is assumed to be constant at $280 \mathrm{~K}$ and the reservoir pressure is kept constant at 150 bar. It is also assumed that flashing does not occur.

\section{DEVELOPMENT OF OBJECTIVE FUNCTION}

A simple notation of the optimization problem is (Edgar et al., 2001):
Minimize $f(x)$
Objective Function
Subject to $h(x) \quad$ Equality constraints
$g(x) \quad$ Inequality constraints

Here $x$ is the vector of decision variables. For the case of the oil field, the decision variables are the flow rates of lift gas through each of the gas lift choke valves.

The amount of oil produced from the reservoir $\left(w_{o}^{i}\right)$ is a function of the amount of lift gas $\left(w_{g a}^{i}\right)$ injected into the well.

$$
w_{o}^{i}=f\left(w_{g a}^{i}\right)
$$

Due to limited supply of lift gas, the sum of the total gas injected into the five oil wells should be equal to that supplied by the compressor. This gives rise to a linear equality constraint as, 


$$
\sum_{i=1}^{5} w_{g a}^{i}=w_{g c}
$$

$w_{g a}^{i}$ is the mass flow rate of lift gas through the gas lift choke valve of $i^{\text {th }}$ well and $w_{g c}$ is the mass flow rate of lift gas supplied by compressor.

In order to find the lower and the upper limit of the lift gas injection rate, it is assumed that the gas lift choke valve of each well will be at least $10 \%$ open and to the maximum $80 \%$ open. This range could have been chosen to be from $0 \%$ (fully closed) to $100 \%$ (fully open), however, seldom are the choke valves operated at their full throttle. Also shutting down one of the oil wells completely would give rise to other factors (for e.g. re-starting the well by following the well unloading procedures). Moreover, the open loop as well as the closed loop simulation results (Sharma et al., 2011) of the simple model of the oil field used in this paper does not show the opening of the valves below $10 \%$ and above $80 \%$ under normal operating conditions. For calculating the gas mass flow rate at $10 \%$ and $80 \%$ opening of the valves, steady state operating condition of the field has been considered. In normal operating condition, the pressure upstream of the gas lift choke valve $\left(P_{c}\right)$ is assumed to be 200 bar and the pressure downstream of the gas lift choke valve $\left(P_{a}^{i}\right)$ is assumed to be 170 bar (this assumption is made after analyzing a year's data from a real oil field).

Mass flow rate through the gas lift choke valve $\left(w_{g a}^{i}\right)$ is obtained by using the standard flow equation developed by Instrument Society of America (ANSI/ISA.S75.01, 1989),

$$
w_{g a}^{i}=\frac{N_{6} C_{v}\left(u_{1}^{i}\right) Y_{1}^{i} \sqrt{\rho_{g p} \max \left(P_{c}-P_{a}^{i}, 0\right)}}{3600} \quad \frac{\mathrm{kg}}{\mathrm{sec}}
$$

$N_{6}=27.3$ is the valve constant, $u_{1}^{i}$ is valve opening of the $i^{\text {th }}$ gas lift choke valve expressed in percentage, $P_{c}$ and $P_{a}^{i}$ are the pressures upstream and downstream of the $i^{\text {th }}$ gas lift choke valve in bars, $\rho_{g p}$ is the density of gas in the distribution pipeline in $\mathrm{kg} / \mathrm{m}^{3}$ which is a function of the upstream pressure $P_{c} . Y_{1}^{i}$ is the gas expansion factor and $C_{v}\left(u_{1}^{i}\right)$ is the valve characteristic as a function of its opening.

We assume the gas expansion factor $\left(Y_{1}^{i}\right)$ to be:

$$
\begin{gathered}
Y_{1}^{i}=1-\alpha_{Y}\left(\frac{P_{c}-P_{a}^{i}}{\max \left(P_{c}, P_{c}^{\min }\right)}\right) \\
\alpha_{Y}=\text { constant }=0.66
\end{gathered}
$$

$P_{c}^{\text {min }}$ is the minimum pressure in the gas distribution pipeline. For $P_{c}=200 \mathrm{bar}, P_{a}^{i}=170 \mathrm{bar}$ and $P_{c}^{\min }$
$=10 \mathrm{bar}$, the value of the gas expansion factor $Y_{1}^{i}=$ 0.901 .

Valve characteristic as a function of its opening $\left(C_{v}\left(u_{1}^{i}\right)\right)$ is modelled by three linear equations as shown in eq. (5). The function in eq. (5) is fitted to the data supplied by the choke supplier.

$$
C_{v}\left(u_{1}^{i}\right)= \begin{cases}0 & u_{1}^{i} \leq 5 \\ 0.111 u_{1}^{i}-0.556 & 5<u_{1}^{i} \leq 50 \\ 0.5 u_{1}^{i}-20 & u_{1}^{i}>50\end{cases}
$$

For $u_{1}^{i}=80 \%, C_{v}(80 \%)=20$. For $u_{1}^{i}=10 \%$, $C_{v}(10 \%)=0.554$

The density of the lift gas in the distribution pipeline $\left(\rho_{g p}\right)$ at $P_{c}=200$ bar can be expressed using the gas law as,

$$
\rho_{g p}=10^{5} \frac{M P_{c}}{\left.R T_{p} z\right|_{P_{c}=200}} \quad \frac{\mathrm{kg}}{\mathrm{m}^{3}}
$$

Here, $M=$ molecular weight of the lift gas $=20 \times$ $10^{-3} \mathrm{~kg}, P_{c}=$ pressure of gas distribution manifold $=$ $200 \mathrm{bar}, R=$ Universal gas constant $=8.314 \mathrm{~kg} / \mathrm{mole}$, $T_{p}=$ gas temperature in the distribution manifold $=$ $280 \mathrm{~K}$ and $\left.z\right|_{P_{c}=200}=$ gas compressibility factor at a pressure of 200 bar.

The gas compressibility factor given by eq. (7) is expressed as a polynomial function of gas pressure $\mathrm{P}$ in bar (assuming constant temperature of $280 \mathrm{~K}$ at the bottom of the sea). It is a curve fitted (LSQ-method) to calculations from PVTsim (PVTsim, 2008) using the lift gas composition and assuming constant temperature.

$z=-2.572 \times 10^{-8} P^{3}+2.322 \times 10^{-5} P^{2}-0.005077 P+1$

For a pressure $P=200$ bar, the gas compressibility factor $z=0.7076$. The density of the lift gas in the distribution pipeline from eq. (6) is then, $\rho_{g p}=242.83$ $\mathrm{kg} / \mathrm{m}^{3}$. Then using eq. (3), the flow rate of the lift gas through the gas lift choke valve is: For $u_{1}^{i}=80 \%$ (gas lift choke valve opening of $80 \%), w_{g a}^{i}=11.66 \mathrm{~kg} / \mathrm{sec}$. For $u_{1}^{i}=10 \%$ (gas lift choke valve opening of $10 \%$ ), $w_{g a}^{i}=0.323 \mathrm{~kg} / \mathrm{sec}$.

Thus the lower and upper bounds for the gas injection rate is given by,

$$
0.323 \leq w_{g a}^{i} \leq 11.66 \quad \frac{\mathrm{kg}}{\mathrm{sec}}
$$

Since the production of the oil from the reservoir is a function of the gas injection rate, let us express $w_{o}^{i}$ as a function of $w_{g a}^{i}$. Flow rate of the lift gas from the annulus into the tubing through the gas injection valve $\left(w_{\text {ginj }}^{i}\right)$ is given by (ANSI/ISA.S75.01, 1989), 


$$
w_{\text {ginj }}^{i}=\frac{K^{i} Y_{2}^{i} \sqrt{\rho_{g a}^{i} \max \left(P_{a i n j}^{i}-P_{t i n j}^{i}, 0\right)}}{3600} \frac{k g}{s e c}
$$

$K^{i}$ is the gas injection valve constant, $P_{a i n j}^{i}$ is the pressure upstream of the gas injection valve in the annulus and $P_{\text {tinj }}^{i}$ is the pressure downstream of the gas injection valve in the tubing, $\rho_{g a}^{i}$ is the average density of gas in the annulus. $Y_{2}^{i}$ is the gas expandability factor given by,

$$
Y_{2}^{i}=1-\alpha_{Y}\left(\frac{P_{a i n j}^{i}-P_{t i n j}^{i}}{\max \left(P_{a i n j}^{i}, P_{a i n j}^{\min }\right)}\right), \alpha_{Y}=0.66
$$

$P_{a i n j}^{\min }$ is the minimum pressure of gas in the annulus at the point of injection. Arranging and solving eq. (9) we get,

$$
P_{\text {tinj }}^{i}=P_{a i n j}^{i}-\frac{\left(\frac{w_{g i n j}^{i} \times 3600}{K^{i} Y_{2}^{i}}\right)^{2}}{\rho_{g a}^{i}} \quad b a r
$$

$P_{a i n j}^{i}$ can also be expressed by adding the hydrostatic pressure drop due to lift gas inside the annulus to the pressure $P_{a}^{i}$ as,

$$
P_{a i n j}^{i}=P_{a}^{i}+\frac{\rho_{g a}^{i} g L_{a \_v l}^{i}}{10^{5}}
$$

$L_{a \quad v l}^{i}$ is the vertical depth of the annulus from the well head to the point of injection in meters. Combining eq. (10) and eq. (11) we get,

$$
P_{t i n j}^{i}=P_{a}^{i}+\frac{\rho_{g a}^{i} g L_{a \_}^{i} v l}{10^{5}}-\frac{\left(\frac{w_{g i n j}^{i} \times 3600}{K^{i} Y_{2}^{i}}\right)^{2}}{\rho_{g a}^{i}}
$$

Similarly arranging and solving eq. (3) we get,

$$
P_{a}^{i}=P_{c}-\frac{\left(\frac{w_{g a}^{i} \times 3600}{N_{6} C_{v}\left(u_{1}^{i}\right) Y_{1}^{i}}\right)^{2}}{\rho_{g p}} \quad b a r
$$

The bottom hole pressure or well flow pressure $P_{w f}^{i}$ is,

$$
P_{w f}^{i}=P_{t i n j}^{i}+\frac{\rho_{o} g L_{r \_v l}^{i}}{10^{5}} \quad b a r
$$

$L_{r \quad v l}^{i}$ is the vertical length of the tubing below the gas injection point up to reservoir opening in meters and $\rho_{o}$ is the density of crude oil in $\mathrm{kg} / \mathrm{m}^{3}$. From eq. (10) and eq. (14) we get,
$P_{w f}^{i}=P_{a}^{i}+\frac{\rho_{g a}^{i} g L_{a \_v l}^{i}}{10^{5}}-\frac{\left(\frac{w_{g i n j}^{i} \times 3600}{K^{i} Y_{2}^{i}}\right)^{2}}{\rho_{g a}^{i}}+\frac{\rho_{o} g L_{r \_v l}^{i}}{10^{5}}$ bar

From eq. (13) and eq. (15) we get,

$$
\begin{aligned}
P_{w f}^{i}=P_{c}- & \frac{\left(\frac{w_{g a}^{i} \times 3600}{N_{6} C_{v}\left(u_{1}^{i}\right) Y_{1}^{i}}\right)^{2}}{\rho_{g p}}+\frac{\rho_{g a}^{i} g L_{a \_v l}^{i}}{10^{5}} \\
& -\frac{\left(\frac{w_{g i n j}^{i} \times 3600}{K^{i} Y_{2}^{i}}\right)^{2}}{\rho_{g a}^{i}}+\frac{\rho_{o} g L_{r_{-} v l}^{i}}{10^{5}} \text { bar }
\end{aligned}
$$

The mass flow rate of crude oil flowing from the reservoir into the tubing $\left(w_{o}^{i}\right)$ is calculated using the PI (Productivity Index) model of the well (American.Petroleum.Institute, 1994; Brown and Beggs, 1977).

$$
w_{o}^{i}=\frac{P I^{i} \max \left(P_{r}^{i}-P_{w f}^{i}, 0\right)}{3600} \quad \frac{k g}{s e c}
$$

$P_{r}^{i}$ is the reservoir pressure which is assumed to be constant at 150 bar. Then from eq. (16) and eq. (17) we get,

$$
\begin{gathered}
w_{o}^{i}=\frac{P I^{i}}{3600}\left(P_{r}^{i}-P_{c}+\frac{\left(\frac{w_{g a}^{i} \times 3600}{N_{6} C_{v}\left(u_{1}^{i}\right) Y_{1}^{i}}\right)^{2}}{\rho_{g p}}-\frac{\rho_{g a}^{i} g L_{a \_}^{i} v l}{10^{5}}\right) \\
+\frac{P I^{i}}{3600}\left(\frac{\left(\frac{w_{g i n j}^{i} \times 3600}{K^{i} Y_{2}^{i}}\right)^{2}}{\rho_{g a}^{i}}-\frac{\rho_{o} g L_{r \_v l}^{i}}{10^{5}}\right) \frac{k g}{\sec } \quad(18)
\end{gathered}
$$

Finally the objective function for the optimization problem considering all the five oil wells of the field can be expressed as,

Maximize

$$
\begin{aligned}
& f\left(w_{g a}^{i}\right)=\sum_{i=1}^{5} \frac{P I^{i}}{3600} \\
& \left(P_{r}^{i}-P_{c}+\frac{\left(\frac{w_{g a}^{i} \times 3600}{N_{6} C_{v}\left(u_{1}^{i}\right) Y_{1}^{i}}\right)^{2}}{\rho_{g p}}-\frac{\rho_{g a}^{i} g L_{a \_}^{i} v l}{10^{5}}\right) \\
& +\sum_{i=1}^{5} \frac{P I^{i}}{3600}\left(\frac{\left(\frac{w_{g i n j}^{i} \times 3600}{K^{i} Y_{2}^{i}}\right)^{2}}{\rho_{g a}^{i}}-\frac{\rho_{o} g L_{r_{-} v l}^{i}}{10^{5}}\right) \frac{k g}{s e c}
\end{aligned}
$$


subject to the linear equality constraint,

$$
\sum_{i=1}^{5} w_{g a}^{i}=w_{g c}
$$

and the bounds

$$
0.323 \leq w_{g a}^{i} \leq 11.66 \quad \frac{\mathrm{kg}}{\mathrm{sec}}
$$

Optimization problem formulated in eqs. (19), (20) and (21) is a non- linear programming with constraints. In this paper, two approaches to solve the optimization problem have been discussed. The first approach is with the use of 'fmincon' solver from MATLAB optimization toolbox and the second approach is with the use of 'hill climbing' method.

\section{OPTIMIZATION USING SOLVER FROM MATLAB OPTIMIZATION TOOLBOX}

'fmincon' is a built-in function in MATLAB for finding the minimum of a constrained non-linear objective function of several variables starting at user specified initial estimate. However, for the case of maximizing oil production, the objective function has to be maximized instead of minimized. This can be achieved by using 'fmincon' to the objective function reflected along the $\mathrm{x}$-axis i.e. by taking the negative of the objective function.

In our case, since we have the bound constraints (eq. (21)) as well as the linear constraints (eq. (20)), 'fmincon' uses sequential quadratic programming (SQP) method with the active set optimization algorithm (MathWorks.Inc., 2011). Moreover, to get the optimal points as close as possible to the global maximum, global search algorithm has been used along with 'fmincon'. The global search algorithm starts the 'fmincon' solver from multiple start points (MathWorks.Inc., 2011). For details about how global search algorithm and the SQP method are implemented in 'fmnicon', refer to the documentation of MATLAB.

To use fmincon, matrices containing coefficients of the linear equalities $\left(A_{e q}\right.$ and $\left.B_{e q}\right)$ and the vectors for the lower and upper bounds ( $l b$ and $u b$ ) have to be passed as argument to it. Eq. (20) can be arranged in matrix form $A_{e q} x=B_{e q}$ as,

$$
\underbrace{\left[\begin{array}{ccccc}
1 & 1 & 1 & 1 & 1
\end{array}\right]}_{A_{e q}} \underbrace{\left[\begin{array}{c}
w_{g a}^{1} \\
w_{g a}^{2} \\
w_{g a}^{3} \\
w_{g a}^{4} \\
w_{g a}^{5}
\end{array}\right]}_{x}=\underbrace{w_{g c}}_{B_{e q}}
$$

Similarly, from eq. (21), the lower and upper bounds are,

$$
\underbrace{0.323}_{l b} \leq w_{g a}^{i} \leq \underbrace{11.66}_{u b}
$$

\subsection{Optimization with the cascade control structure}

Optimization of the lift gas distribution in a gas lifted oil well is performed along with the cascade control structure. A schematic diagram of the cascade control structure is shown in Figure 4. In cascade control strategy, a pressure transducer measures the pressure of the common gas distribution manifold which is then taken as feedback to a pressure controller. The set point to this pressure controller is 200 bar. The output of the pressure controller gets added to the nominal set points of five flow controllers. The result is then given as the current set point to the five flow controllers.

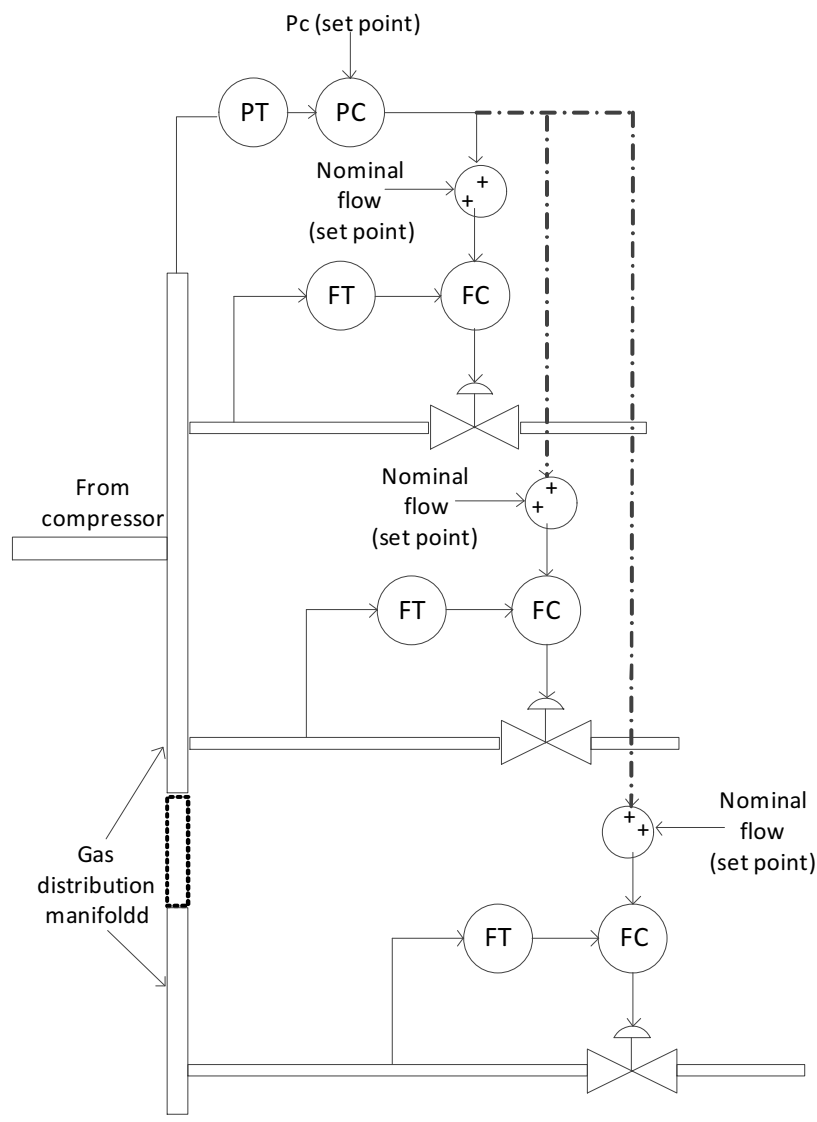

Figure 4: Schematic for cascade control structure

The five flow controllers control the rate of flow of lift gas through each of the gas lift choke valves. The pressure controller is responsible for maintaining a fairly 
constant pressure of the lift gas in the gas distribution manifold by manipulating proper lift gas flow rates through the valves. The optimal flow rate set points calculated by the optimization algorithm is actually utilized by the cascade control structure as the nominal flow set points for five flow controllers.

\subsection{Discussion on simulation results}

For all the simulation results presented in the paper, the process parameters ( $P I, K$ etc.) were calculated by using the data from a real oil field. Similarly, the physical parameters like the lengths, cross sectional areas etc. of different pipelines of the real oil field have been used for simulation. From the real field data, it was observed that well 5 produced the least (about 150 $\mathrm{Sm}^{3} / \mathrm{hr}$ of crude oil) and well 4 produced the highest (about $452 \mathrm{Sm}^{3} /$ hr of crude oil). The Productivity Indices of the five oil wells followed the relation as shown in eq. (24) (Sharma et al., 2011).

$$
P I^{4}>P I^{1}>P I^{2}>P I^{3}>P I^{5}
$$

The non-linear objective function with linear equality constraints and inequality bounds was solved using MATLAB optimization toolbox. It was assumed that the total available lift gas at normal operating condition was $40,000 \mathrm{Sm} 3 / \mathrm{hr}$. At first the available lift gas was distributed randomly (non-optimally) among well 1 to well 5 consuming $15 \%, 17 \%, 25 \%, 23 \%$ and $20 \%$ of the total flow respectively.

The process was first allowed to reach the steady state with the controllers running alone. In such a case, the controllers manipulate the gas flow rates among the oil wells in a way that the gas pressure in the common gas distribution manifold is kept constant at 200 bar. The total oil produced from the oil field with nonoptimal gas distribution is shown in Figure 5 .

In Figure 5, the total oil produced from the five oil wells without any optimization was about $332.5 \mathrm{~kg} / \mathrm{sec}$ which actually resembled to the total oil production of the real oil field at Norne.

However, after the process reached the steady state, at $\mathrm{t}=50$ hours, the optimizer loop was activated once to find the optimal set points for the flow controller. The values of the different process variables (like pressure of gas distribution manifold, valve openings, densities etc.) used in the objective function (eq. (19)) were taken as the steady state values. The optimal set points returned by the optimizer were used as the new nominal gas flow rate set points for the cascade control structure (see Figure 4). The simulation was then continued with the controllers working alone again.

After using the new optimal set points, the total oil produced from the oil field was increased by $14 \mathrm{~kg} / \mathrm{sec}$

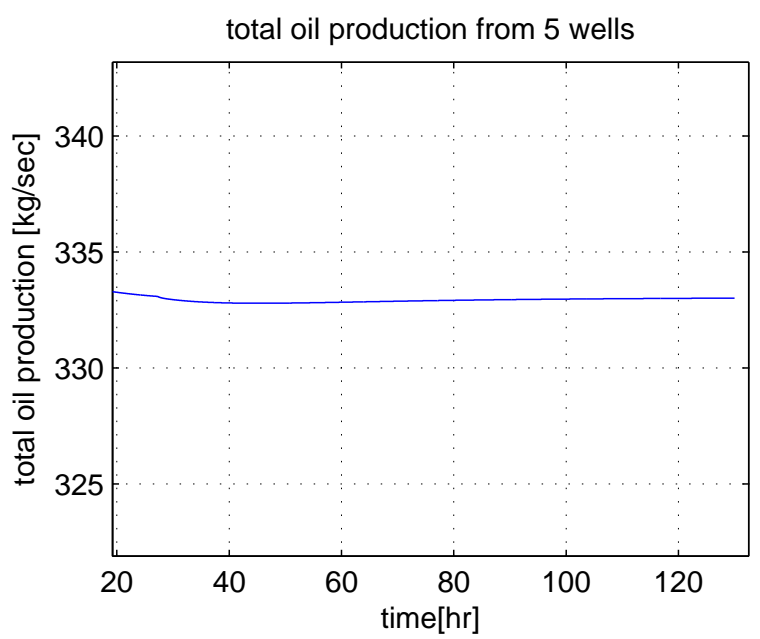

Figure 5: Total oil production without optimization

from about $332.5 \mathrm{~kg} / \mathrm{sec}$ (non-optimal production) to about $346.5 \mathrm{~kg} / \mathrm{sec}$ (optimal production) as shown in Figure 6.

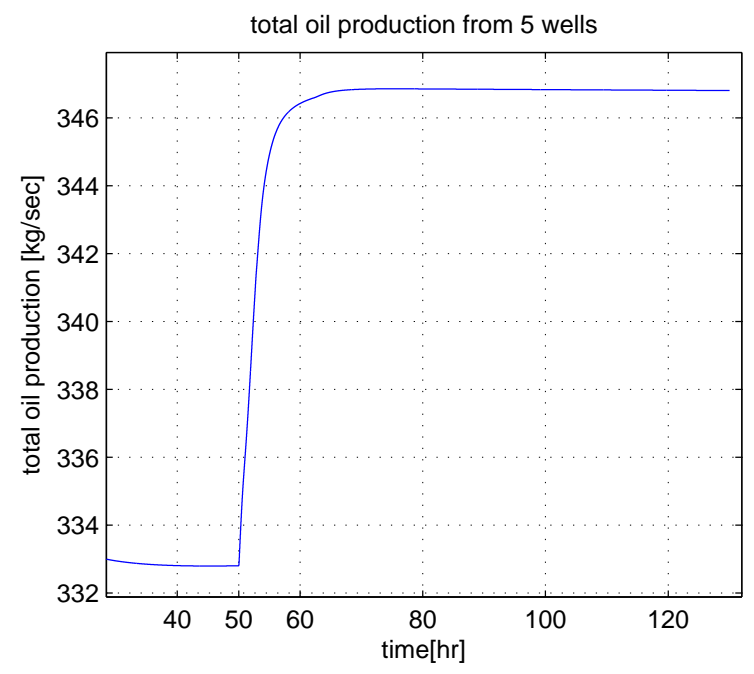

Figure 6: Total oil production with optimization turned 'ON' at $\mathrm{t}=50$ hours

The distribution of the lift gas through the choke valves without any optimization is shown in Figure 7 .

As can be seen from Figure 7, when the optimizer was not activated at $\mathrm{t}=50$ hours after the process reached the steady state, there was no re-distribution of the lift gas and the process continued to operate in its steady state producing a total of $332.5 \mathrm{~kg} / \mathrm{sec}$ of crude oil.

However, when the optimizer was turned 'ON' at $\mathrm{t}$ $=50$ hours, due to the new optimal set points of the flow controllers generated by the optimizer, the lift gas distribution among the oil well was changed. The re- 


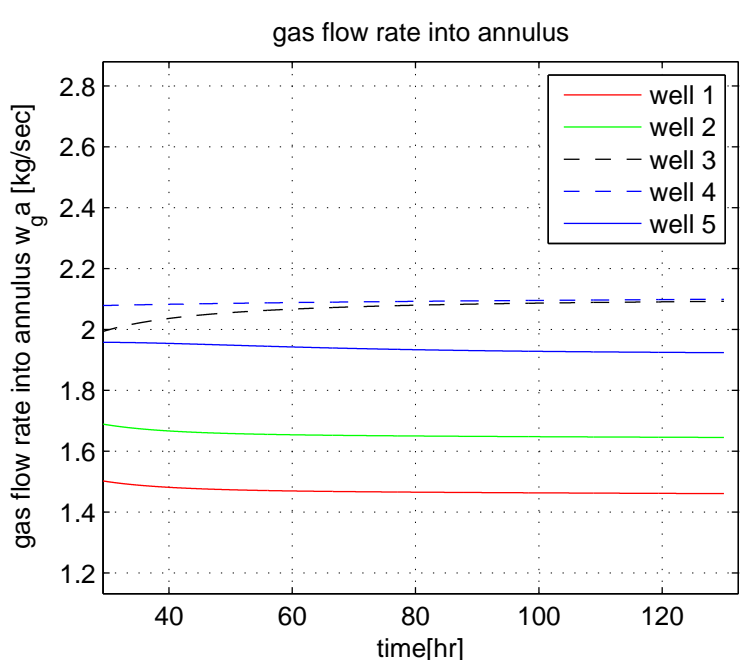

Figure 7: Lift gas distribution among wells without optimization

distribution of the lift gas through the five gas lift choke valve after the application of the new optimal flow set points and with the controllers still active is shown in Figure 8.

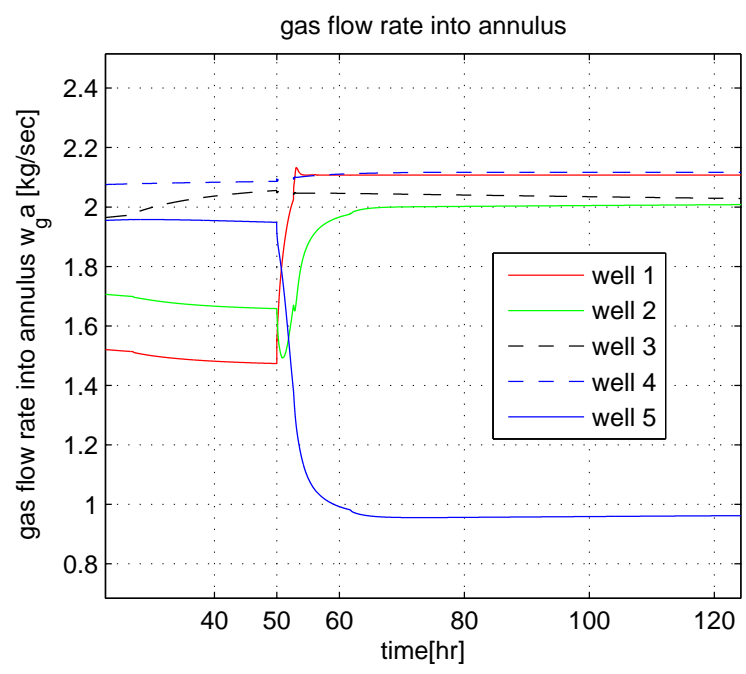

Figure 8: Lift gas distribution among wells with optimization

The optimizer allocated the least amount of lift gas to oil well 5 which in fact has the smallest Productivity Index value. However, gas injection into well 1 and well 2 were increased. Well 1 and well 2 both have higher Productivity Index value than well 5 (see eq. (24)). Due to the re- distribution of the lift gas, the oil produced from individual oil wells was also changed in accordance to the change in the distribution of the lift gas as shown in Figure 9.

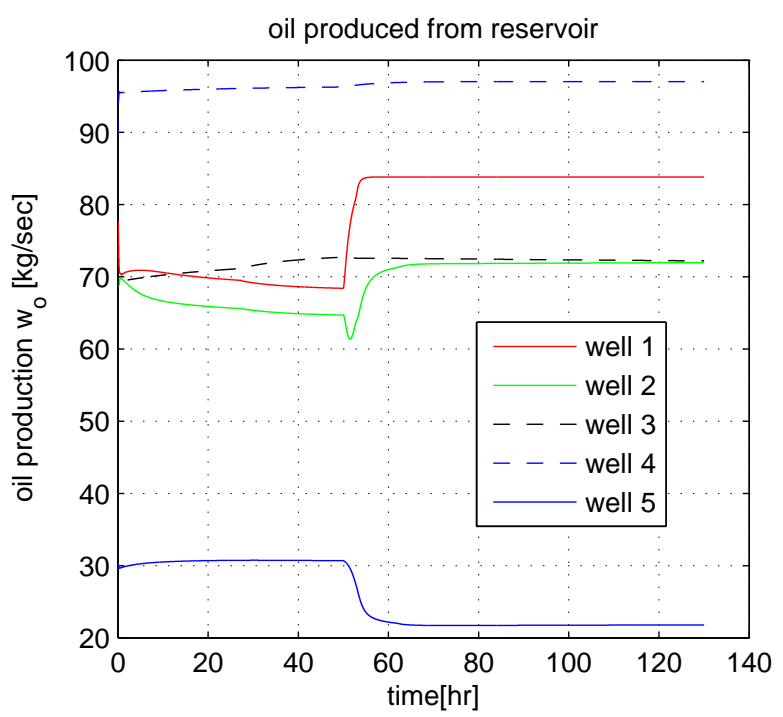

Figure 9: Oil produced from each oil well after optimization

Production of oil from oil well 1 and oil well 2 was increased because the amount of lift gas supplied to these wells was increased after optimization (see Figure 8). Similarly, the oil flow rate from well 5 was decreased due to less injection of lift gas after optimization (see Figure 9). Finally, it can be concluded from the simulation results that the outcome of optimization using the built-in solver in MATLAB is an increased total oil production. Expressing it in percentage, the increased production due to process optimization was $4.21 \%$.

\subsection{How often to perform optimization?}

The effect on the total oil production when optimization is performed multiple times under the presence of input disturbance is an interesting topic to discuss. To study this, optimization was performed at $\mathrm{t}=50$ hours under the availability of $40000 \mathrm{Sm}^{3} / \mathrm{hr}$ of gas supply (see Figure 10). The process with increased oil production due to this first place optimization was then allowed to reach the steady state. After the total oil production flow rate reached steady state, at $\mathrm{t}=90$ hours, the supply of lift gas was reduced to $36000 \mathrm{Sm}^{3} / \mathrm{hr}$ (application of input disturbance). Due to the reduction in supply of gas, the total oil production started to decrease as was expected from the process dynamics. The process was again allowed to reach the steady state at this lower supply of lift gas. Then at $\mathrm{t}=120$ hours, when the total oil production flow rate reached steady state, optimization loop was activated for the second time.

Figure 10 shows that when the optimization was activated for the second time after the application of input 
disturbance, it had no effect and the total oil production rate remained the same. The initial values given to the optimizer loop turned ON at $\mathrm{t}=120$ hours were the steady state flow rate values through each gas lift choke valves. The optimizer loop after performing calculations returned back the same initial values as the optimal values.

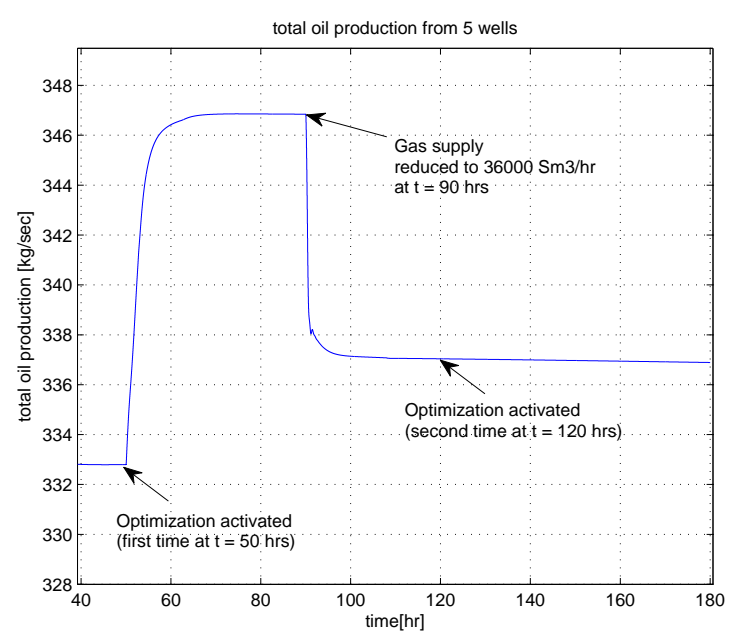

Figure 10: Total oil production when optimization was performed for the second time after input disturbance

This could be due to the combined effect of the optimizer activated for the first time at $\mathrm{t}=50$ hours and the cascade control structure. The process may have already obtained the optimal flow rate set points for all the five flow controllers when the optimizer was activated for the first time. When input disturbance was given at $\mathrm{t}=90$ hours, the controllers may have decreased the gas flow rates through the valves in an optimal way because of the already available optimal flow rate set points. So the reduced total production due to reduced gas supply may have already acquired the optimal steady state at $\mathrm{t}=120$ hours.

\subsection{Optimization after the reduction of the supply of lift gas}

An obvious question that can probably arise is the doubt of whether the optimizer loop failed to function properly for a reduced supply of lift gas. To justify this question about behaviour of the optimizer activated for the second time, an interesting point would be to see how the total oil production will be affected when the optimizer is activated for the first time only after the application of the input disturbance i.e. without any previous activation of the optimizer. In this case, at $\mathrm{t}=60$ hours when the process reaches steady state for $40000 \mathrm{Sm}^{3} / \mathrm{hr}$ supply of gas, instead of activating the optimizer, the gas supply was reduced to 36000 $\mathrm{Sm}^{3} / \mathrm{hr}$. The reduced oil production due to reduced supply of gas was again allowed to reach the steady state. Then at $\mathrm{t}=120$ hours, the optimizer was activated for the first time.

As can be seen from Figure 11, when the optimizer was activated for the first time after applying the input disturbance without any prior optimizations, the total production of the oil increased by around $15 \mathrm{~kg} / \mathrm{sec}$ from about $322 \mathrm{~kg} / \mathrm{sec}$ to about $337 \mathrm{~kg} / \mathrm{sec}$. This clearly implies that the optimizer loop functions properly even for reduced supply of gas.

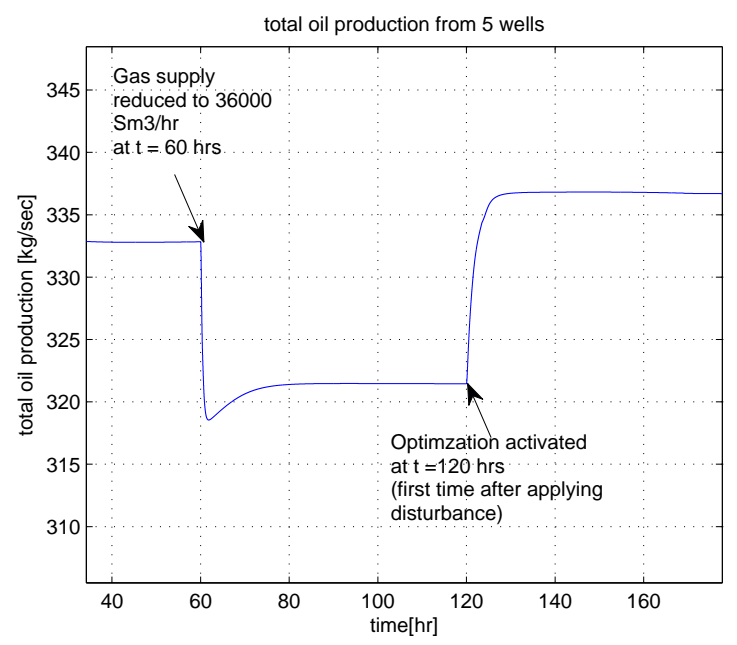

Figure 11: Total oil production when optimization was performed for the first time, only after input disturbance was applied

Optimal total oil production for the reduced supply of the lift gas was about $337 \mathrm{~kg} / \mathrm{sec}$ for both the cases:

a) When the optimizer was activated for the second time after the application of the input disturbance (see Figure 10)

b) When the optimizer was activated for the first time after the application of the input disturbance (see Figure 11)

This suggests that one time single optimization is sufficient enough to bring the process to optimal operating condition and further optimization is not necessary when changing total gas supply as input disturbance. In other words, the cascade control structure along with the steady state optimizer behaves as a selfoptimizing control scheme for the optimal distribution of the available lift gas among multiple oil wells in a gas lifted oil field.

However, it should be noted that in this paper we have considered the supply of the lift gas the only disturbance present in the system. Behaviour of the pro- 
posed self-optimizing control scheme when other additional parameters are considered as disturbance to the system has not been considered here and is left as future improvement works.

\section{OPTIMIZATION USING HILL CLIMBING METHOD}

Hill Climbing is an iterative method of finding the maximum/minimum of a function $f(x)$ with the decision variable(s) $x$ where only one of the decision variables is changed at a time keeping all the others unchanged. Iteration is started with the initial values of the decision variables provided by the user. The algorithm then tries to find better value of $f(x)$ by increasing/decreasing only a single decision variable at one time. If the change provides a better solution than before, the decision variable is slightly incremented and the new solution is calculated. Hill climbing aims to ascend to a peak by repeatedly moving to an adjacent state with a higher fitness (Juels and Wattenberg, 1994). This step is repeated until the increment of the decision variable provides no further improved solution. The whole process is repeated for each remaining decision variable. Finally, the set of decision variables $\mathrm{x}$ is the optimal set of variables.

However, one difficulty in using hill climbing method in the case of the oil field where the decision variables are the gas flow rate through the five gas lift choke valves is that, when an increment is made in the gas flow rate of only one of the gas lift choke valve, gas flow rate through another (or all of the remaining 4 valves) has to be decreased exactly by the same amount by which the increment was made to maintain the total gas flow rate constraint (the total supply gas flow rate should be equal to the sum of the gas flow rates through the five gas lift choke valves). Since at least two decision variables have to be manipulated at the same time, this approach can be considered to be a modified version of the general hill climbing method. Furthermore, the hill climbing method for solving the optimization problem has been done to check the performance of the optimization problem solved by using 'fmincon' solver of MATLAB optimization toolbox as described in Section 4.

\subsection{Strategy for hill climbing method}

The gas lift choke valves are assumed to be never fully closed and never fully open. They are assumed to be open from $10 \%$ to $80 \%$. If the supply gas flow rate is denoted by 'total flow' then $10 \%$ gas lift valve opening of one of the well means that amount of gas flowing through that well is $3.5 \%$ of 'total flow' and that for
$9.22 \mathrm{~kg} / \mathrm{sec}$ gas flow rate (total gas flow rate at normal condition) through the well, it means that the amount of gas flowing through the well is $100 \%$ of 'total flow'.

Initially all the five oil wells have an equal lift gas distribution of $20 \%$ of 'total flow'. The strategy is to decrease the flow rate in one of the wells (also called here as 'starting well') from initial equal distribution of $20 \%$ of 'total flow' to $3 \%$ of 'total flow' in smaller steps of $1 \%$ of 'total flow' at each iteration. At the same time the flow rate of another well (also called here as 'helping well') is increased from $20 \%$ of 'total flow' to $37 \%$ of 'total flow' with the same time step of $1 \%$ of 'total flow' at each iteration. 18 iterations are performed and the total oil production at each iteration is calculated and stored. After this, keeping the same oil well as 'starting well', the remaining three oil wells perform the role of the 'helping well' turn by turn. A sub-total of 72 iterations will be performed and for each iteration, the total oil production is calculated and stored.

When all the remaining three oil wells have completed working as 'helping well', the role of the 'starting well' is undertaken by the next oil well. The remaining oil wells will again function as 'helping well' for this new 'starting well'. The whole process is repeated until each of the five oil wells works as 'starting well'. At the end, a total of 360 iterations will be performed. The set of gas flow rates which gives the highest oil production among these 360 iterations is considered to be the optimal gas flow rates.

It should be noted that the step change of $1 \%$ of 'total flow' is equivalent to $5 \%$ change in the flow rate of lift gas in each well. This step of $5 \%$ change in the lift gas flow rate in each well is assumed to provide observable change in the total oil production. Moreover, for each oil well, the iteration swings from $3 \%$ of total production to $37 \%$ of total production, which is sufficient enough to provide a set of good local optimal set points.

\subsection{Discussion on simulation results}

Hill climbing method was used to solve the non-linear objective function with linear equality constraints and inequality bounds. It was assumed that the total available lift gas was $40,000 \mathrm{Sm}^{3} / \mathrm{hr}$ at normal operating conditions. At first the total available lift gas was distributed randomly (non-optimally) among well 1 to well 5 consuming $15 \%, 17 \%, 25 \%, 23 \%$ and $20 \%$ of the total available lift gas respectively.

The cascade control structure of Figure 4 was used along with the optimizer for optimal control and distribution of lift gas and for optimal production of oil. All the assumptions and conditions used for optimization using built-in 'fmincon' solver from MATLAB op- 
timization toolbox have also been used for hill climbing method.

Starting with some non-optimal gas distribution, the process was first allowed to reach the steady state with the controllers running alone. At $\mathrm{t}=60$ hours, when the process reached the steady state, the optimizer loop was activated once to find the optimal set points for the five flow controllers. The optimal set points returned by the optimizer were used as the new nominal set points for the cascade control structure. The simulation was then continued with the controllers alone. After the availability of new nominal set points, the total oil produced from the oil field was increased to about $347 \mathrm{~kg} / \mathrm{sec}$ as shown in Figure 12.

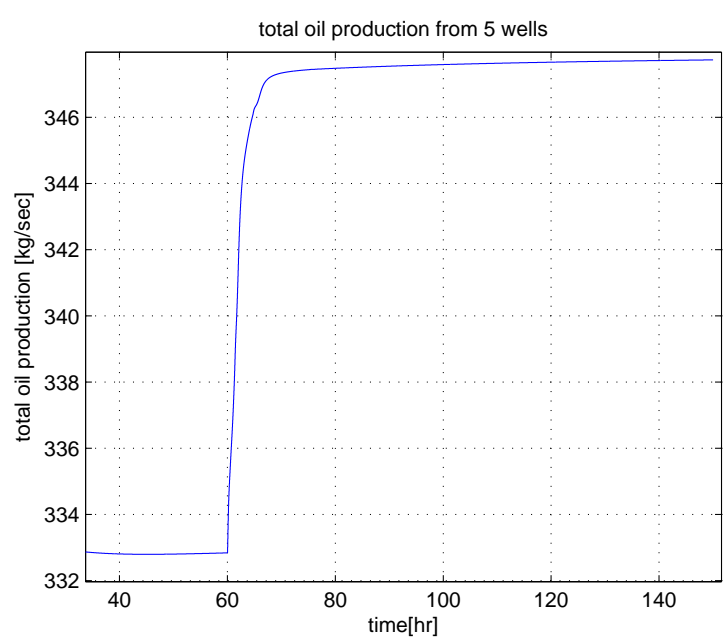

Figure 12: Total oil production with hill climbing optimization turned 'ON' at $\mathrm{t}=60$ hours

Without any optimization, the total oil production was about $332 \mathrm{~kg} / \mathrm{sec}$ as shown in Figure 5. So the hill climbing optimization caused an increase in the total oil production by about $15 \mathrm{~kg} / \mathrm{sec}$. When expressed in percentage, the oil production was increased by about $4.5 \%$, which is very much similar to what was obtained with optimization using solver from MATLAB optimization toolbox.

The distribution of lift gas among the five gas lift choke valves changed after the application of the new optimal flow set points and with the controller still active as shown in Figure 13.

The gas flow rate though well 5 which is the least producing well was decreased. For well 1 and well 2 which have relatively higher $P I$ values than well 5 , the gas flow rates was increased after the hill climbing optimizer was turned 'ON' at $\mathrm{t}=60$ hours. Without any optimization, there was no re-distribution of the lift gas among the wells and the process continued to operate in its steady state as shown in Figure 7 with

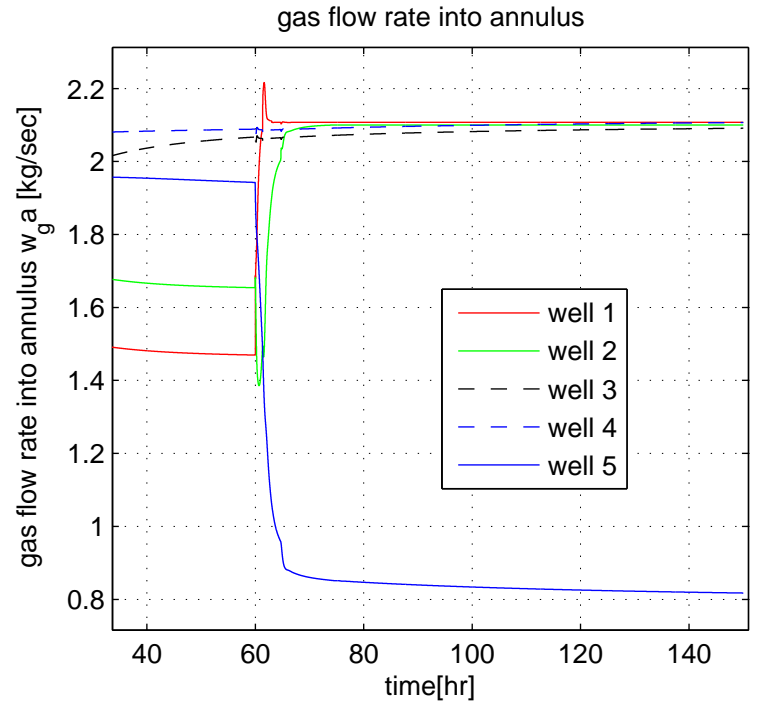

Figure 13: Gas distribution among wells using hill climbing optimizer turned 'ON' at $\mathrm{t}=60$ hours

non-optimal oil production. Since re-distribution of the lift gas took place at $\mathrm{t}=60$ hours as a result of optimization, the oil produced from individual oil wells were also changed in accordance to the distribution of the lift gas as shown in Figure 14.

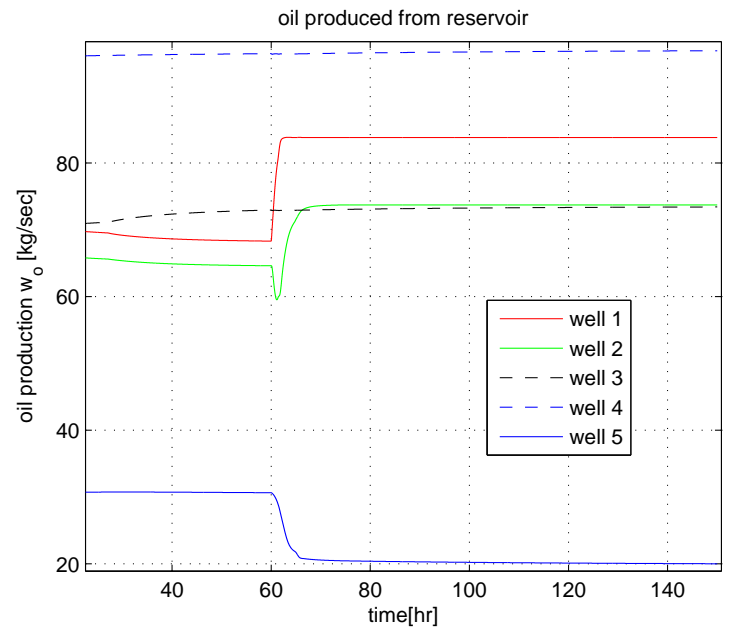

Figure 14: Oil produced from each oil well after optimization with hill climbing method

Production of oil from oil well 1 and oil well 2 increased because the lift gas supplied to these wells was increased after optimization (see Figure 13). Similarly, the oil flow rate from well 5 decreased due to less injection of lift gas after optimization (see Figure 14) which is very similar to what was obtained using the built-in 
solver of MATLAB optimization toolbox.

Finally, it can be concluded from the simulation results that the outcome of optimization using the hill climbing method is an increase in the total oil production. The simulation results obtained using the hill climbing method can also be used as the verification of the results obtained from built-in optimization solver.

\section{COMPARISON OF THE TWO OPTIMIZATON METHODS}

Both the methods of optimization were successfully tested through simulations and both of them could optimize the operation of the lift gas distribution resulting in increased oil production. The amount of total oil production increased after the optimization was almost the same for both the methods. So it is difficult to compare these two methods based on how much oil production was increased by each optimization method.

The built-in solver of MATLAB optimization toolbox uses the mathematical model of the oil field. Mathematical model of a complex process is just an approximation of the real process. Models of complex process will always have some assumptions and it cannot represent the real process completely. Moreover, as time passes and as the process becomes older, the dynamics of the process may change over time due to several factors. The mathematical model developed and tested when the plant was young may not be a good representation of the older model. Under this situation, optimization methods based on the mathematical model of the process might not at all provide optimal results in the real oil fields. Thus hill climbing optimization procedure which is independent on mathematical modeling of the process is advantageous in this regard over the use of MATLAB solver for solving an optimization problem which is an important strength for real applications.

'fmincon' which is a built-in solver of Optimzation toolbox in MATLAB uses active set optimization algorithm utilizing sequential quadratic programming (MathWorks.Inc., 2011). This method uses the information of the gradient of each decision variables such that all the decision variables can be changed in each iteration. In case of hill climbing method, only a single variable can be altered at a time (the other variable(s) were changed only to meet the constraints). Also, the built-in solver uses 'global solution' class which has a tendency to provide better optimal solution (more towards global solution) than the hill climbing method which only provides local optimal solution.

A number of field experiments should be performed if hill climbing method is to be applied to real oil field. The normal operation of the existing oil field has to be disrupted for performing these tests during which time there might be some loss of total production of oil. However, this loss of oil during the test period (if they are not very long) can be compromised with an increase in the total oil production for a longer period of time due to optimization. To conclude, the use of 'fmincon' as built-in solver from MATLAB optimization toolbox is recommended when a good mathematical model of the process is available for optimization. But, for real oil fields, the hill climbing method might be more beneficial and realistic to use.

\section{CONCLUSION}

For optimal distribution of the available lift gas among the five oil wells in order to maximize the total oil production, a non-linear optimization problem with linear constraints and inequality bounds was formulated using the model of the process at steady state. The optimization problem was then solved using two methods; one was using the MATLAB optimization toolbox and another was by using the hill climbing method of optimization. Both the optimization methods could increase the total oil production by about $4 \%$. One advantage of doing optimization with hill climbing method in a real oil field is that it does not require a mathematical model of the oil field so it is free of modeling errors and assumptions. However, it does require a number of experiments to be performed in the real field for which the normal operation of the oil field might have to be obstructed.

Optimization with the 'fmincon' solver was performed including the global search algorithm, so this method has the tendency to provide better optimal solution (more towards global solution) than the hill climbing method which only provides local optimal solution. Assuming the supply of lift gas as the input disturbance, the cascade control structure along with the steady state optimizer functions as self-optimizing control structure.

\section{References}

American.Petroleum.Institute. API gas lift manual, Book 6 of the Vocational Training series. Exploration and Production Department, Washington DC 2005-4070, USA, third edition edition, 1994.

ANSI/ISA.S75.01. Flow Equations for Sizing Control Valves, Standards and Recommended Practices for Instrumentation and Control, 10th editoin, vol 2 edition, 1989. 
Brown, K. E. and Beggs, H. D. The technology of artificial lift methods, Volume 1, Inflow Performance, Multiphase flow in pipes, The flowing well. PennWell Publishing Company, Tulsa, Oklahoma, ISBN: 0-87814-031-X, 1977.

Camponogara, E. and Nakashima, P. Solving a gaslift optimization problem by dynamic programming. European Journal of Operational Research, 2006. 174:1220-1246. doi:10.1016/j.ejor.2005.03.004.

Edgar, T. E., Himmelblau, D. M., and Lasdon, L. S. Optimization of chemical processes. McGraw-Hill Chemical Engineering Series, second edition edition, 2001.

Juels, A. and Wattenberg, M. Stochastic hill climbing as a baseline method for evaluating genetic algorithms. Dept. of Computer Science and Dept. of Mathematics, University of California at Berkeley, 1994.

Kosmidis, V. D., Perkins, J., and Pistikopoulos, E. N. A mixed integer optimization formulation for the well scheduling problem on petroleum fields. Journal of Computers and Chemical Engineering, 2005. 29:1523-1541. doi:10.1016/j.compchemeng.2004.12.003.

MathWorks.Inc. R2011a documentation - optimization toolbox [online]. Available at: http://www.mathworks.com/help/toolbox/optim /ug/fmincon.html (Accessed: 20 May 2011), 2011.

PVTsim. PVT simulation program developed for reservoir engineers, flow assurance specialists, PVT lab engineers and process engineers [online]. Available at: http://www.pvtsim.com (Accessed: 20 January 2011), 2008.
Rashid, K. Optimal allocation procedure for gaslifted optimization. Industrial and Engineering Chemistry Research, 2010. 49:2286-2294. doi:10.1021/ie900867r.

Ray, T. and Sarker, R. Genetic algorithm for solving a gas lift optimization problem. Journal of Petroleum Science and Engineering, 2007. 59:84-96. doi:10.1016/j.petrol.2007.03.004.

Saepudin, D., Soewono, E., Sidarto, K., Gunawan, A., Siregar, S., and Sukarno, P. An investigation on gas lift performance curve in an oil producing well. International Journal of Mathematics and Mathematical Science, 2007. 2007:1-15. doi:10.1155/2007/81519.

Sharma, R., Fjalestad, K., and Glemmestad, B. Modeling and control of gas lifted oil field with five oil well. In 52nd International Conference of Scandinavian Simulation Society, SIMS 2011, Sep 29-30. Västerås, Sweden, pages 47-59, 2011.

Souza, J. N. M., Medeiros, J. L., Costa, A. L. H., and Nunes, G. C. Modeling, simulation and optmization of continuous gas lift systems for deepwater offshore petroleum production. Journal of Petroleum Science and Engineering, 2010. 72:277289. doi:10.1016/j.petrol.2010.03.028.

Sukarno, P., Saepudin, S., Soewono, E., Sidarto, K., and Gunawan, A. Optimization of gas injection allocation in a dual gas lift well system. Journal of Energy Resources Technology, 2009. 131(3). doi:10.1115/1.3185345.

Zhong, H., Li, Y., and Liu, Y. An approach for optimization of gas-lift allocation to a group of wells and oil field. In 3rd International Symposium of National Key Laboratory for Oil and Gas Reservoir Exploitation. Chengu, China, pages 1-11, 2004. 